\title{
Analysis of Cocaine and Crack Cocaine via Thin Layer Chromatography Coupled to Easy Ambient Sonic-Spray Ionization Mass Spectrometry
}

\author{
Bruno D. Sabino ${ }^{1,2^{*}}$, Wanderson Romão ${ }^{3}$, Morena L. Sodré ${ }^{1}$, Deleon N. Correa ${ }^{3}$, \\ Denise B. Rocha Pinto ${ }^{1}$, Fábio O. M. Alonso ${ }^{1}$, Marcos N. Eberlin ${ }^{3}$ \\ ${ }^{1}$ Institute of Criminalistic Carlos Éboli, Rio de Janeiro, Brazil, \\ ${ }^{2}$ National Institute of Metrology, Standardization and Industrial Quality, Av. N. Sra. das Graças, \\ Duque de Caxias, Brasil \\ ${ }^{3}$ ThoMSon Mass Spectrometry Laboratory, Institute of Chemistry, University of Campinas, \\ Campinas, Brazil \\ E-mail: *brsabino@ig.com.br
}

Received June 20, 2011; revised July 27, 2011; accepted August 3, 2011

\begin{abstract}
Cocaine and crack cocaine are usually seized with a great diversity of adulterants, such as benzocaine, lidocaine, caffeine, and procaine. The forensic identification of cocaine in these drug mixtures is normally performed using colorimetric testing kits, but these tests may suffer from interferences providing false-positive or false-negatives. In this work, we describe the use of thin layer chromatography coupled to easy sonic-spray ambient ionization mass spectrometry (TLC/EASI-MS) for rapid and secure analysis of cocaine and crack cocaine. Fifteen cocaine samples were analyzed, and all of them revealed positive TLC/EASI-MS results for cocaine, but other drugs and adulterants were also detected such as lidocaine, caffeine, benzocaine, lactose, benzoylecgonine, and ecgonidine. False positives and false negatives, as judged by the TLC Rf values, were identified via on-spot characterization by EASI-MS. The TLC/EASI-MS combination seems therefore to provide an appropriate technique for secure forensic investigations of illicit drugs.
\end{abstract}

Keywords: Cocaine; Crack, TLC, Illicit Drug, EASI-MS

\section{Introduction}

Cocaine is an illicit drug produced from the leaves of Eritroxylum coca normally via extraction with organic solvents followed by purification, liquid-liquid extraction and a final conversion from free base cocaine to hydrochloride cocaine [1]. Crack is a combination of cocaine hydrochloride, baking soda, and other adulterants that form a rock-like substance [2]. Figure 1 shows a picture of typical powder cocaine (left) and crack cocaine (right) samples seized by the Rio de Janeiro State Police.

Street drugs are subject to many procedures of adulteration and dilution. To imitate its effects, adulterants are often molecules with similar pharmacological, sensorial and physical-chemical properties as those of the main drug. Diluents are organic or inorganic compounds with no significant pharmacological properties, intentionally added to the street-drug sample to increase the volume and weight of the final product [3]. Illicit samples of cocaine are rarely pure. Figure 2 shows the chemical structure of cocaine Figure 2(a), its main impurities that arise via the manufacturing process such as benzoylecgonine Figure 2(b), cinnamoylcocaine Figure 2(c), and benzoic acid Figure 2(d), adulterants such as the anesthetics lidocaine Figure 2(f), procaine Figure 2(g) and benzocaine Figure 2(h) and other central nervous system (CNS) ac-

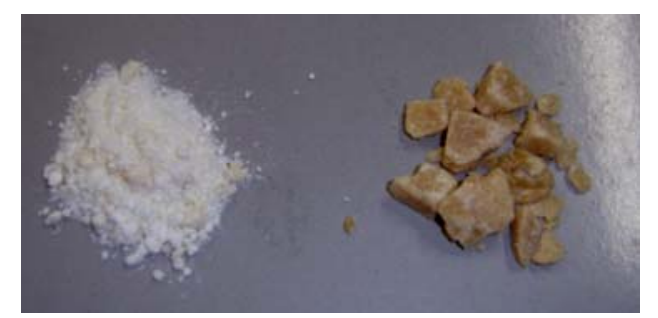

Figure 1. Typical samples of powder (left) and crack (right) cocaine. 

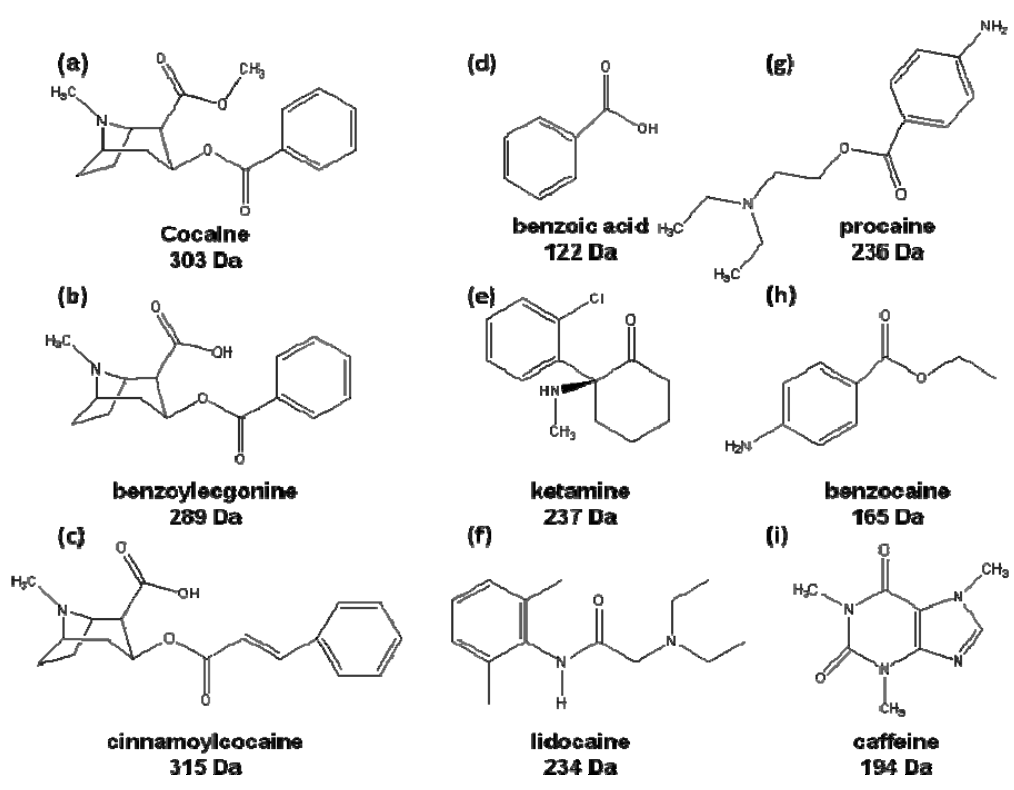

Figure 2. Structures of molecules normally found in cocaine samples.

tive drugs, such as ketamine Figure 2(e) and caffeine Figure 2(i) that are usually present in addition or in substitution of cocaine in illegal drug formulations.

The identification of cocaine and crack cocaine is often performed by forensic laboratories using the Scott Ruybal test [4] that employs a reagent kit to develop a blue color when cocaine is present. The colorimetric tests are the gold standard for forensic analysis of controlled substances, and offer a reasonably reliable means for very rapid screening. These tests display, however, poor specificity, and may sometimes provide false-positives or false-negatives, specially for the more complex mixtures or impure samples and for common adulterants such as lidocaine and ketamine [5].

In forensic analysis, thin layer chromatography (TLC) is also a classic, simple, and versatile method for drug analysis. TLC is limited, however, in terms of secure characterization and is therefore often used in parallel with other methods of structural analysis. Quantitation is normally not attempted in TLC, but direct ultraviolet densitometric measurements of TLC spots have been used to quantify components found in illicit drug samples [1].

Recently, a new set of ambient ionization mass spectrometric ionization techniques have been introduced. These techniques allow desorption, ionization and characterization of analytes directly from surfaces or natural matrixes [6]. These methods, known collectively as ambient MS methods, have become also attractive alternatives in forensic analysis since they require no sample preparation or pre-separation. Key examples of these techniques are desorption electrospray ionization (DESI) [1-4,7-11], direct analysis in real time (DART) [8-12], extractive electrospray ionization (EESI), desorption at- mospheric-pressure photoionisation (DAPPI), atmospheric solids analysis probe (ASAP) [13], desorption atmospheric pressure chemical ionization (DAPCI), electrospray-assisted laser desorption ionization (ELDI) [14], and easy ambient sonic spray ionization (EASI) [15].

Among these ionization/desorption techniques, EASI is one of the simplest, gentlest and most easily implemented [15]. An EASI source operates with no voltages, radiation, discharges or heating and can be constructed and installed in a few minutes from simple MS laboratory parts. EASI is assisted only by compressed $\mathrm{N}_{2}$ (or air) used for sonic spraying [16] that creates very small droplets from the solvent, which end up being charged due to statistical imbalanced distribution of cations and anions in these minute droplets (Figure 3). The dense stream of the sonic charged droplets is directed to the sample surface, where desorption and further transference of charge to analytes molecules occurs. EASI-MS can also operate in the Venturi easy ambient sonic-spray ionization (V-EASI) mode with the additional benefit of solution self pumping [17] and has already been successfully applied to several forensic investigations such as analysis of ecstasy [18] and mCPP tablets [19], inks [20], chemical fingerprinting of banknotes [21], perfumes [22] and LSD blotters [23]. In this work, the ability of TLC/EASI-MS to analyze cocaine and crack cocaine street samples was tested.

\section{Experimental}

\subsection{Reagents and Samples}

HPLC grade methanol and formic acid were obtained 


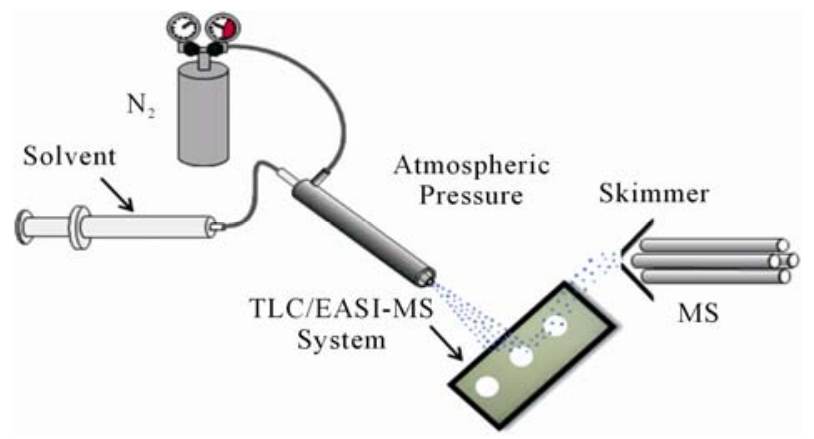

Figure 3. Schematics of the TLC/EASI-MS coupling used to directly analyze cocaine and crack cocaine samples.

from Merck. Eight samples of cocaine white powder and seven of crack cocaine were provided by the Rio de Janeiro State Civil Police. Cocaine, caffeine, lidocaine, benzocaine, procaine, and ketamine standards solutions (1 $\mathrm{mg} / \mathrm{mL}$ ) were purchased from Radian (Austin, TX, USA).

\subsection{Sample Preparation}

The investigated drug samples were seized by the police in Rio de Janeiro, Brazil, during the years of 2008-2009 and were received by the Carlos Éboli Criminalistic Institute of Rio de Janeiro Civil State Police for analysis. Crack cocaine rocks were pulverized and cocaine white powder samples were homogenized and $10 \mathrm{mg}$ of each sample was dissolved in $10 \mathrm{ml}$ of methanol. After centrifugation, the upper layer was transferred to a glass vial and analyzed by TLC/EASI-MS.

\subsection{TLC Procedure}

Precoated plates (silica gel 60 GF 254, Merck, 6100 Darmstadt, Germany) were used in all cases. The plates were dried for $30 \mathrm{~min}$ at $80^{\circ} \mathrm{C}$ and then stored in a desiccator. The sample solution of each seized drug and aliquots of the standards solutions $(3 \mu \mathrm{L})$ were applied to silica gel plates. These plates were then developed in an horizontal chamber (Camag, Switzerland). The developing distance was $8 \mathrm{~cm}$. Two mobile phases were tested: 1) methanol, chloroform and acetic acid (20:75:5 v\%), and 2) acetone. After development, the plates were dried at $100^{\circ} \mathrm{C}$ for $15 \mathrm{~min}$. Spots were detected and marked under ultraviolet (UV) radiation at $254 \mathrm{~nm}$.

\subsection{Limit of Detection}

The limit of detection (LOD) of cocaine in TLC plates was set as the minimum concentration that could be visualized with an acceptable level of precision of $\leq 15 \%$ and accuracy of $\pm 15 \%$. LOD samples were analyzed as they were unknown samples in 10 replicates.

\subsection{EASI(+)-MS Procedures}

Experiments were performed on a mono-quadrupole mass spectrometer (LCMS-2010EV-Shimadzu Corp., Japan) equipped with a home-made EASI source (Figure 4), which has been described in details elsewhere [16]. Acidic methanol $\left(0.1 \%\right.$ in volume, $\left.20 \mu \mathrm{L} \cdot \mathrm{min}^{-1}\right)$ and compressed $\mathrm{N}_{2}$ at a pressure of $100 \mathrm{psi}$ were used to form the sonic spray. The capillary-surface and surface-entrance angles were of $45^{\circ}$. Spectra were accumulated for $10 \mathrm{~s}$.

\subsection{Gas Chromatography-Mass Spectrometry (GC-MS) Analysis}

The GC-MS analyses were conducted using a Thermo Scientific (Austin, Texas) Focus gas chromatograph coupled with an ITQ 700 Thermo mass selective detector. The mass spectra scan rate was 3 scans s$~^{-1}$. The GC was operated in splitless mode with a carrier gas (helium grade 5) flow rate of $1.5 \mathrm{~mL} \cdot \mathrm{min}^{-1}$.

The mass spectrometer was operated using $70 \mathrm{eV}$ electron ionization (EI) and a source temperature of $250^{\circ} \mathrm{C}$. The GC injector was maintained at $250^{\circ} \mathrm{C}$ and the transfer line at $250^{\circ} \mathrm{C}$. EI-MS were subjected to background subtraction and averaged using ca. five scans. The samples analyzed were diluted in HPLC grade methanol to give a final concentration of $1 \mathrm{mg} \cdot \mathrm{mL}^{-1}$ and $1 \mu \mathrm{L}$ was introduced via manual injection as individual solutions. The GC temperature program used consisted of an initial temperature of $130^{\circ} \mathrm{C}$ for $1 \mathrm{~min}$ then increased to $280^{\circ} \mathrm{C}$ at $17^{\circ} \mathrm{C} \cdot \mathrm{min}^{-1}$ and held for $11 \mathrm{~min}$. GC/MS was used to confirm all impurities identified by TLC/EASI-MS. All the plates used in the work were prepared in duplicate. The spots shown in the figures were also scratched from a duplicate plate and analyzed by GC-MS.

\section{Results and Discussion}

To demonstrate the applicability of TLC/EASI-MS for

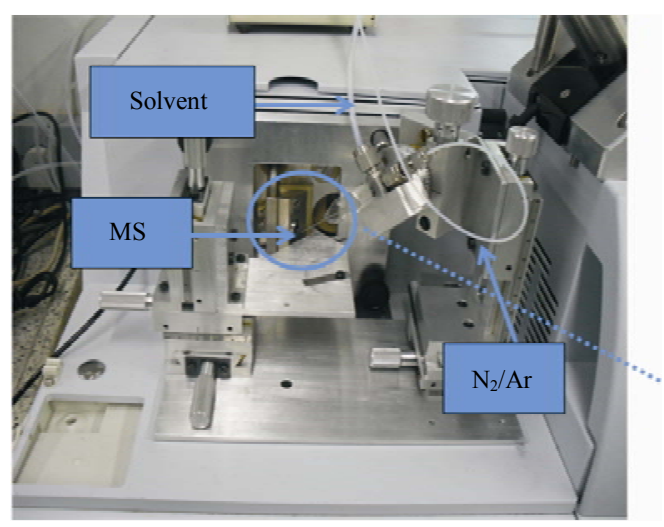

Figure 4. Picture of the EASI-MS system. 
the screening of street samples of cocaine and crack cocaine, we first evaluated the TLC performance using two different eluents and standards solutions, as well as seized drug samples (Figure 5), three of cocaine ( $\operatorname{coc} 1, \operatorname{coc} 3$ and $\operatorname{coc} 6$ ) and four of crack cocaine (crack 2, crack 4, crack 5 and crack 7). Table 1 lists the Rf values obtained. TLC with acetone as mobile phase (Figure 5(a)) showed spots tailing for most powder-cocaine and crack cocaine samples and for some of the standard solutions of cocaine and procaine. The cocaine and procaine spots showed also too close $\mathrm{Rf}$ values $(\mathrm{Rf} \approx 0.45$ and 0.36 , respectively). Therefore, due to these poor results with acetone, a second mobile phase was tested.

TLC with methanol:chloroform:acetic acid (20:75:5 $\checkmark \%$ ) as mobile phase (Figure 5(b)) was more efficient then with acetone since well separated and defined spots for most of the samples were observed, and with the presence of new spots with higher Rf values, interpreted as indicative of the separation of impurities from the cocaine and crack cocaine samples. Additionally, this TLC eluent permitted proper separation and resolution of cocaine standard from others standards (see $\mathrm{Rf}$ values in Table 1). Hence, for TLC/EASI-MS measurements, TLC was performed with methanol:chloroform:acetic acid $(20: 75: 5 v \%)$ as the eluent.

Figures 6(a)-(f) shows the TLC/EASI-MS data for the spots of all standards used. Caffeine and benzocaine showed the lowest ionization efficiency and somewhat poor but still a detectable signal/nose ratio. The great polarity of caffeine and benzocaine is likely to increase their retention by the silica surface decreasing therefore the desorption efficiency via EASI. Similar results were observed to TLC analysis of seized ecstasy tablets by EASI-MS [18].

Figure 7(a) shows the TLC/EASI-MS data for the cocaine spot of a cocaine sample (coc-1).

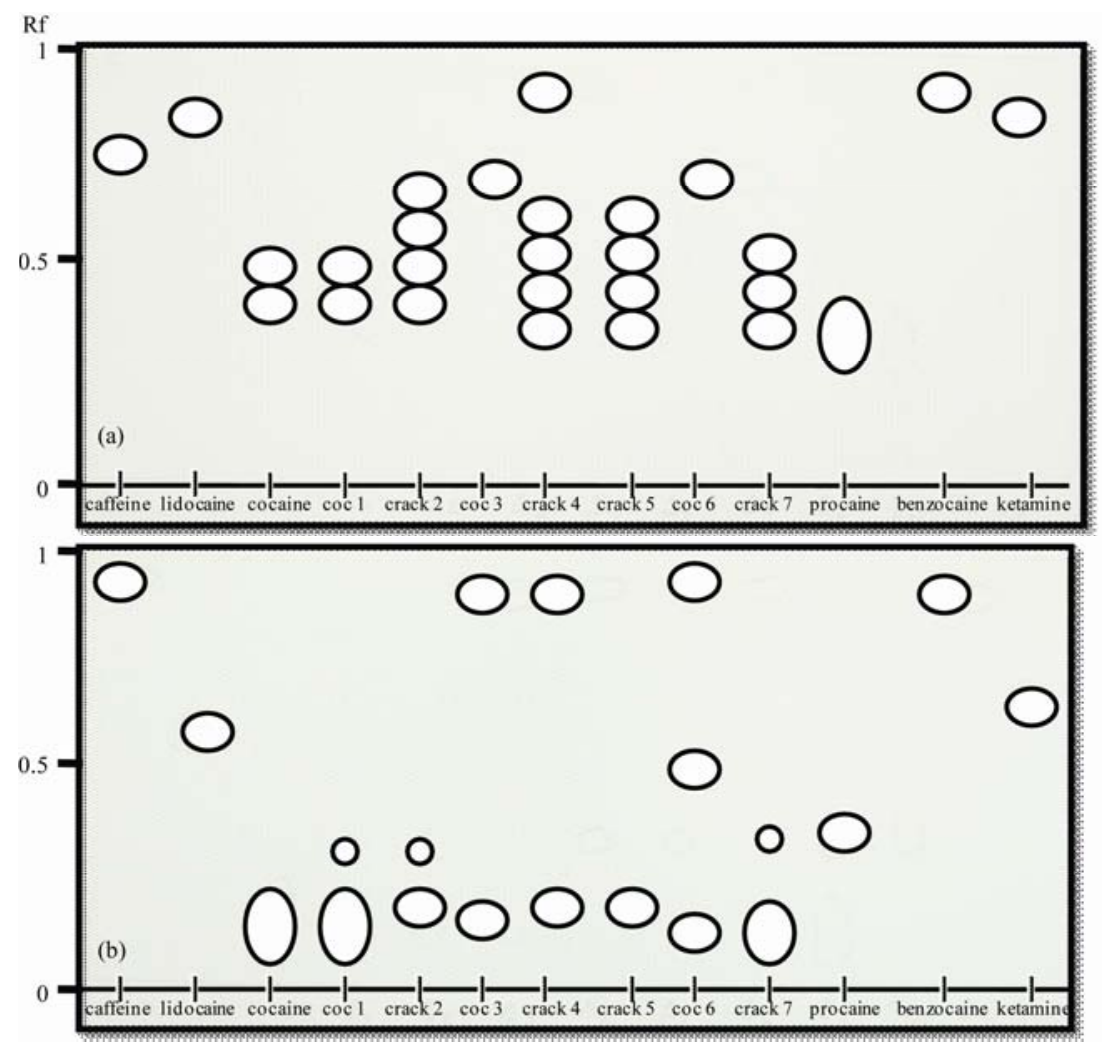

Figure 5. TLC of standards, cocaine samples and crack cocaine samples using acetone (upper), and methanol: chloroform:acetic acid (20:75:5 v\%) (lower) as the mobile phase.

Table 1. Rf values of standards.

\begin{tabular}{ccc}
\hline Compound & Acetone & methanol:chloroform: acetic acid (20:75:5 $\boldsymbol{v} \%)$ \\
\hline Caffeine & 0.75 & 0.94 \\
Lidocaine & 0.83 & 0.47 \\
Cocaine & 0.45 & 0.15 \\
Procaine & 0.36 & 0.36 \\
Benzocaine & 0.92 & 0.89 \\
Ketamine & 0.80 & 0.64 \\
\hline
\end{tabular}



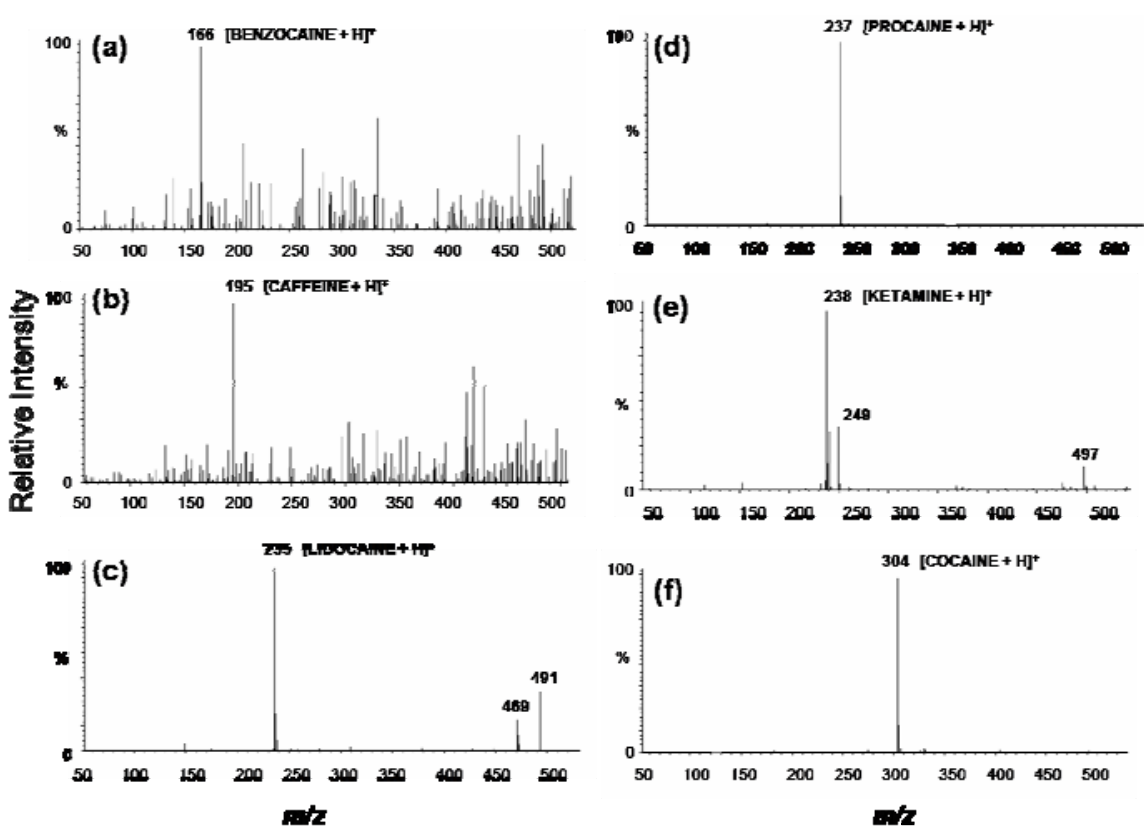

Figure 6. TLC EASI-MS data for standards of: (a) benzocaine, (b) caffeine, (c) lidocaine, (d) procaine, (e) ketamine and (f) cocaine.

Figures 8(a)-(b) show EASI-MS data for the two spots for sample coc-3 with quite distinct $R f$ values.

Figures 9(a)-(b) show EASI-MS data for two spots corresponding to coc- 6 .

Figure 10 shows composite TLC chromatograms of 5 cocaine samples $(\operatorname{coc} 9, \operatorname{coc} 10, \operatorname{coc} 12, \operatorname{coc} 13$ and $\operatorname{coc} 15)$ and 3 crack cocaine samples (crack 8 , crack 11 and crack 14).

The difference in Rf values from the TLC spots of lidocaine standard in coc 10 could be explained by the fact that lidocaine concentration in coc 10 is much lower than the cocaine concentration. This difference in concentrations between these substances may alter the interactions degree between lidocaine, the mobile phase and the stationary silica phase of TLC plates, increasing its RF value, when comparing to the lidocaine spot in standard solution. This result shows the importance of confirming the TLC results with the application of the EASI-MS analysis.

Figures 11(a)-(c) show the EASI-MS data for coc-10 sample.

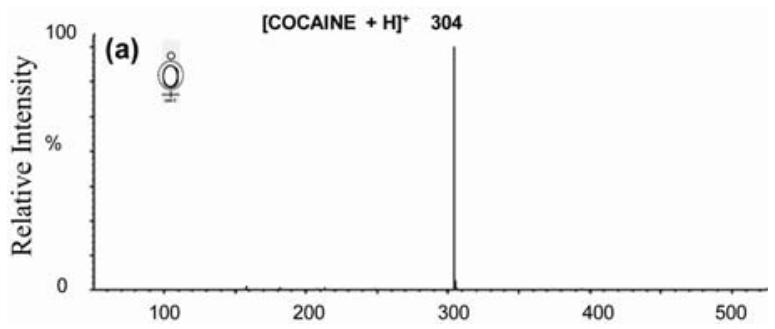

Figure 7. TLC EASI-MS data for the cocaine spot of sample coc-1. PRECISA APAGAR O (a).

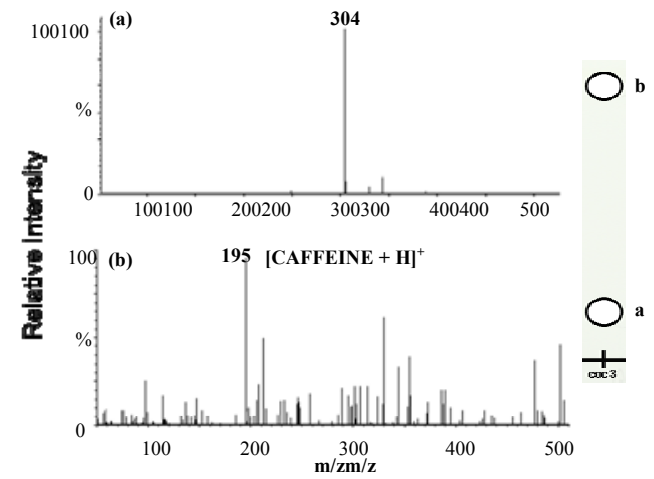

Figure 8. TLC EASI-MS data for two spots of coc 3. Spot (b) is identified as cocaine via its $[\mathrm{M}+\mathrm{H}]^{+}$of $\mathrm{m} / \mathrm{z} 304$ whereas spot a is characterized as caffeine via its $[\mathrm{M}+\mathrm{H}]^{+}$of $\mathrm{m} / \mathrm{z} 195$.

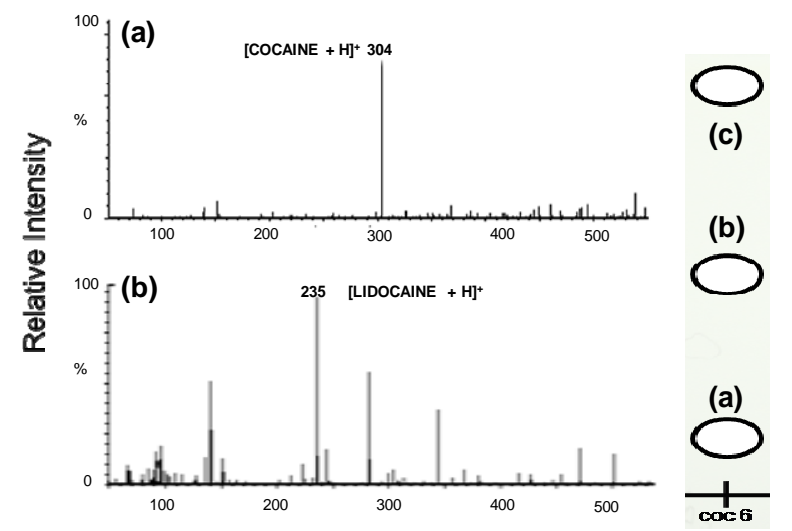

Figure 9. TLC EASI-MS data for spots (b) and (c) of coc-6. Spot $b$ was identified as cocaine and spot a as lidocaine, in agreement with the TLC results (Figure 5(b)). 


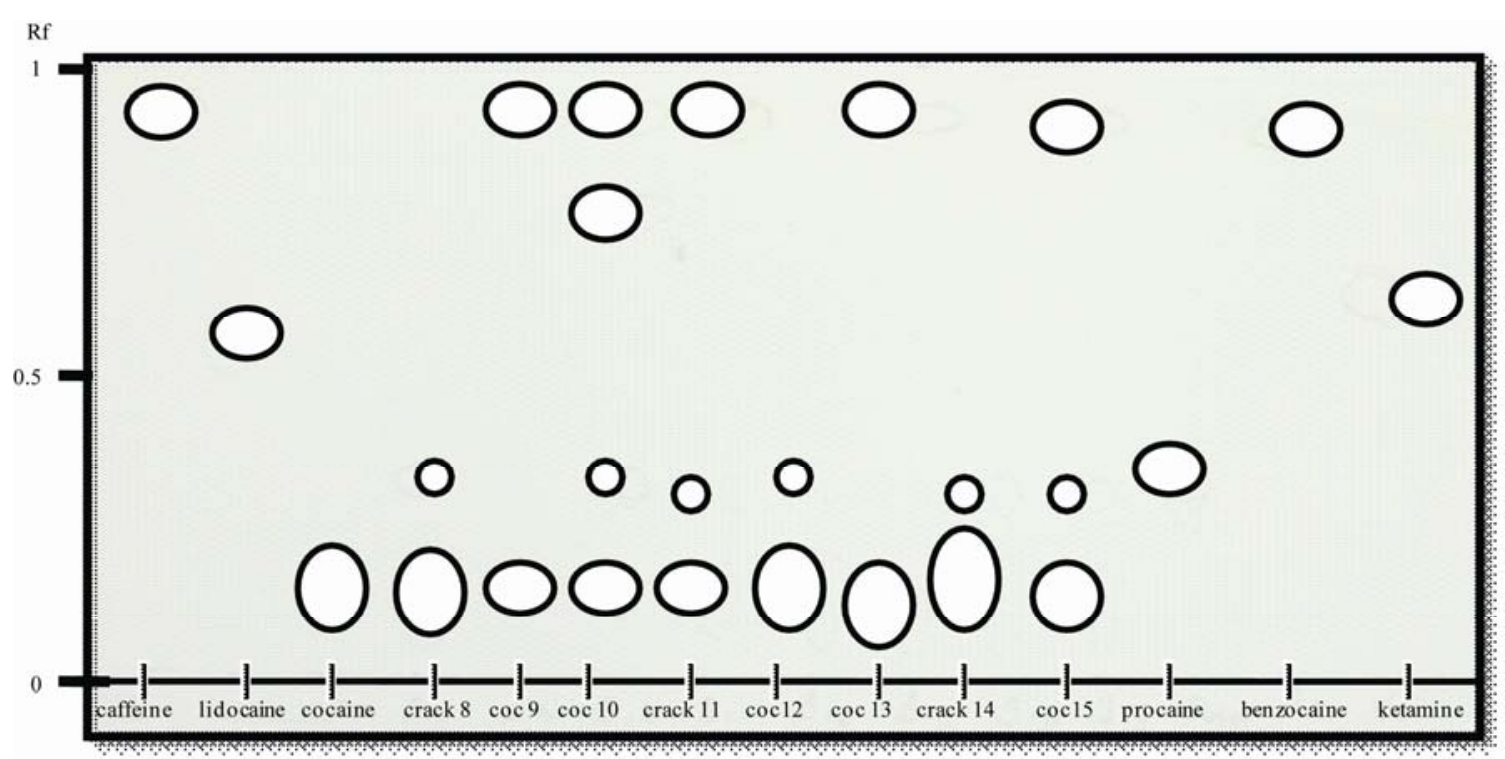

Figure 10. TLC of standards and for 5 cocaine and 3 crack samples. Again, all samples show spots with Rf ( 0.15) coincident with that of cocaine, providing therefore a rapid (but not unequivocally) positive results for cocaine. The EASI-MS data confimed, however, the presence of cocaine. The coc-9, coc-10, crack 11, coc-13 and coc-15 samples showed spots with $R f \approx 0.92$ that points to either caffeine or benzocaine. The coc-10 sample presented the highest number of spots (four). Except for the coc-9 sample, all samples displayed a spot $\mathrm{Rf}$ values of $\approx 0.33$ that points to procaine.

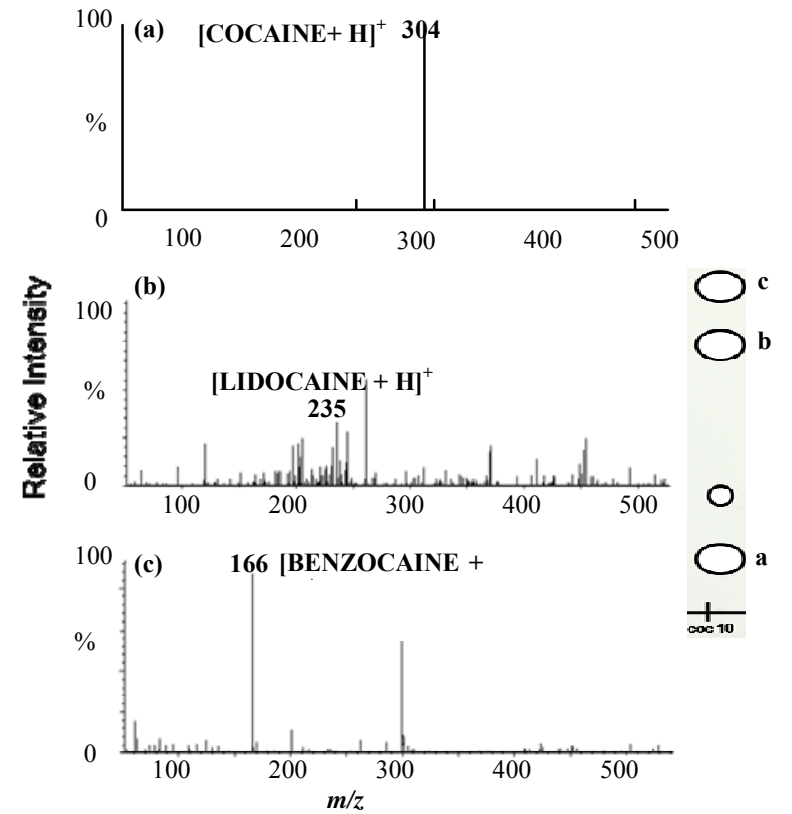

Figure 11. TLC EASI-MS data on spots for coc-10 with the identification of three spots as cocaine $\left([\mathrm{M}+\mathrm{H}]^{+}\right.$of $\left.\mathrm{m} / \mathrm{z} 304\right)$, lidocaine $\left([\mathrm{M}+\mathrm{H}]^{+}\right.$of $\left.\mathrm{m} / \mathrm{z} 235\right)$ and benzocaine $\left([\mathrm{M}+\mathrm{H}]^{+}\right.$of $\mathrm{m} / \mathrm{z}$ 166) confirming the TLC assignments.

\section{Limit of Detection}

The limit of detection found for cocaine in TLC plates was $2 \mu \mathrm{g}$. This result was obtained by the analysis of 10 replicates of cocaine standard solutions, and the spots related to this alkaloid were observed in all the plates.
This limit of detection is quite sufficient to permit its use for routine analysis of cocaine and crack cocaine seized samples in forensic laboratories.

\section{Conclusions}

The validation of TLC results of drug analysis in forensic investigations is crucial to generate unquestionable results and to eliminate false negatives and positives. TLCmethod is rapid, simple and low cost, demanding only basic laboratory equipments and glassware, and is normally applied to screen for cocaine and other active components in seized cocaine and crack cocaine forensic samples. False positive or negatives TLC results may be obtained, however, particularly for samples with impurities of more complex formulations. We have demonstrated that EASI-MS seems to provide a simple and fast tool in forensic analysis able to perform on-spot characterization at the molecular level.

The combination of TLC with eventual EASI-MS inspection seems to provide a powerful combination for forensic investigation of illicit drugs reducing the risks of false positives and false negatives.

\section{Acknowledgments}

We thank financial support from the Brazilian Science foundations CNPq, FAPESP, FINEP and Inmetro. The authors thank the Rio de Janeiro Civil Police for provid- 
ing the cocaine and crack cocaine samples.

\section{References}

[1] M. Chiarotti and N. Fucci, "Comparative Analysis of Heroin and Cocaine Seizures," Journal of Chromatography B: Biomedical Sciences and Applications, Vol. 733, No. 1-2, 1999, pp. 127-136. doi:10.1016/S0378-4347(99)00240-6

[2] S. E. Lankenau, M. C. Clatts, L. A. Goldsamt and D. L. Welle, "Crack Cocaine Injection Practices and HIV Risk: Findings from New York and Bridgeport," Journal of Drug Issues, Vol. 34, No. 2, 2004, pp. 319-332.

[3] D. G. de Carvalho and A. F. Mídio, "Quality of Cocaine Seized in 1997 in the Street-Drug Market of São Paulo City, Brazil," Brazilian Journal of Pharmaceutical Sciences, Vol. 39, No. 1, 2003, pp. 71-75.

[4] R. F. X. Klein and P. A. Hays, "Detection and Analysis of Drugs of Forensic Interest, 1992-2001. A Literature Review," Microgram Journal, Vol. 1, No. 1-2, 2003, pp. 55-153.

[5] W. Jeffrey, "Colour Tests," In: A. Moffat, et al., Ed., Clarke's Analysis of Drugs and Poisons in Pharmaceuticals, Body Fluids and Postmortem Material, Pharmaceutical Press, London, 2004.

[6] D. R. Ifa, L. M. Gumaelius, L. S. Eberlin, N. E. Manicke and R. G. Cooks, "Forensic Analysis of Inks by Imaging Desorption Electrospray Ionization (DESI) Mass Spectrometry," Analyst, Vol. 132, No. 5, 2007, pp. 461-467. doi: $10.1039 / \mathrm{b} 700236 \mathrm{j}$

[7] Z. Takats, J. M. Wiseman, B. Gologan and R. G. Cooks, "Mass Spectrometry Sampling under Ambient Conditions with Desorption Electrospray Ionization," Science, Vol. 306, No. 5695, 2004, pp. 471-473. doi:10.1126/science.1104404

[8] L. A. Leuthold, et al., "Desorption Electrospray Ionization Mass Spectrometry: Direct Toxicological Screening and Analysis of Illicit Ecstasy Tablets," Rapid Communications in Mass Spectrometry, Vol. 20, No. 2, 2006, pp. 103-110. doi: $10.1002 / \mathrm{rcm} .2280$

[9] J. M. Wells, et al., "Implementation of DART and DESI Ionization on a Fieldable Mass Spectrometer," Journal of the American Society for Mass Spectrometry, Vol. 19, No. 10, 2008, pp. 1419-1424. doi:10.1016/j.jasms.2008.06.028

[10] H. Chen, N. N. Talaty, Z. Takats and R. G. Cooks, "Desorption Electrospray Ionization Mass Spectrometry for High-Throughput Analysis of Pharmaceutical Samples in the Ambient Environment," Analytical Chemistry, Vol. 77, No. 21, 2005, pp. 6915-6927. doi:10.1021/ac050989d

[11] T. J. Kauppila, et al., "Desorption Electrospray Ionization Mass Spectrometry for the Analysis of Pharmaceuticals and Metabolites," Rapid Communications in Mass Spectrometry, Vol. 20, No. 3, 2006, pp. 387-392. doi: $10.1002 / \mathrm{rcm} .2304$
[12] R. B. Cody, J. A. Laramee and H. D. Durst, "Versatile New Ion Source for the Analysis of Materials in Open Air under Ambient Conditions," Analytical Chemistry, Vol. 77, No. 8, 2005, pp. 2297-302. doi: $10.1021 / \mathrm{ac} 050162 \mathrm{j}$

[13] C. N. McEwen, R. G. McKay and B. S. Larsen, "Analysis of Solids, Liquids, and Biological Tissues Using Solids Probe Introduction at Atmospheric Pressure on Commercial LC/MS Instruments," Analytical Chemistry, Vol. 1, No. 77, 2005, pp. 7826-7831. doi:10.1021/ac051470k

[14] M. Z. Huang, H. J. Hsu, J. Y. Lee, J. Jeng and J. Shiea, "Direct Protein Detection from Biological Media through Electrospray-Assisted Laser Desorption Ionization/Mass Spectrometry," Journal of Proteome Research, Vol. 5, No. 5, 2006, pp. 1107-1116. doi:10.1021/pr050442f

[15] R. Haddad, R. Sparrapan and M. N. Eberlin, "Desorption Sonic Spray Ionization for (High) Voltage-Free Ambient Mass Spectrometry," Rapid Communications in Mass Spectrometry, Vol. 20, No. 19, 2006, pp. 2901-2905. doi: $10.1002 / \mathrm{rcm} .2680$

[16] A. Hirabayashi, M. Sakairi and H. Koizumi, "Sonic Spray Mass Spectrometry," Analytical Chemistry, Vol. 67, No. 17, 1995, pp. 2878-2882. doi:10.1021/ac00113a023

[17] V. G. Santos, et al., "Venturi Easy Ambient Sonic-Spray Ionization," Analytical Chemistry, Vol. 83, No. 4, 2011, pp. 1375-1380. doi:10.1021/ac102765z

[18] B. D. Sabino, et al., "Analysis of Street Ecstasy Tablets by Thin Layer Chromatography Coupled to Easy Ambient Sonic-Spray Ionization Mass Spectrometry," Brazilian Journal of Analytical Chemistry, Vol. 1, 2010, pp. 6-12.

[19] W. Romao, et al., "Chemical Profile of Meta-Chlorophenylpiperazine (m-CPP) in Ecstasy Tablets by Easy Ambient Sonic-Spray Ionization, X-Ray Fluorescence, Ion Mobility Mass Spectrometry and NMR," Analytical and Bioanalytical Chemistry, Vol. 400, No. 9, 2011, pp. 3053-3064.

doi:10.1007/s00216-011-4883-9

[20] P. M. Lalli, et al., "Fingerprinting and Aging of Ink by Easy Ambient Sonic-Spray Ionization Mass Spectrometry," Analyst, Vol. 135, No. 4, 2010, pp. 745-750. doi: $10.1039 / \mathrm{b} 923398 \mathrm{a}$

[21] L. S. Eberlin, et al., "Instantaneous Chemical Profiles of Banknotes by Ambient Mass Spectrometry," Analyst, Vol. 135, No. 10, 2010, pp. 2533-2539. doi:10.1039/c0an00243g

[22] R. Haddad, R. R. Catharino, L. A. Marques and M. N. Eberlin, "Perfume Fingerprinting by Easy Ambient Sonic-Spray Ionization Mass Spectrometry: Nearly Instantaneous Typification and Counterfeit Detection," Rapid Communications in Mass Spectrometry, Vol. 22, No. 22, 2008, pp. 3662-3666. doi:10.1002/rcm.3788

[23] W. Romão, et al., "LSD and 9,10-Dihydro-LSD Analyses in Street Drug Blotter Samples via Easy Ambient Sonic-Spray Ionization Mass Spectrometry (EASI-MS)," Journal of Forensic Sciences, in press. 